\title{
Realization of Coherent Optically Dense Media via Buffer-Gas Cooling
}

\section{Citation}

Hong, Tao, Alexey V. Gorshkov, David Patterson, Alexander S. Zibrov, John M. Doyle, Mikhail D. Lukin, and Mara G. Prentiss. 2009. Realization of coherent optically dense media via buffer-gas cooling. Physical Review A 79(1): 013806.

\section{Published Version}

doi:10.1103/PhysRevA.79.013806

\section{Permanent link}

http://nrs.harvard.edu/urn-3:HUL.InstRepos:5349609

\section{Terms of Use}

This article was downloaded from Harvard University's DASH repository, and is made available under the terms and conditions applicable to Other Posted Material, as set forth at http:// nrs.harvard.edu/urn-3:HUL.InstRepos:dash.current.terms-of-use\#LAA

\section{Share Your Story}

The Harvard community has made this article openly available.

Please share how this access benefits you. Submit a story.

\section{Accessibility}




\title{
Realization of coherent optically dense media via buffer-gas cooling
}

\author{
Tao Hong, ${ }^{1,2}$ Alexey V. Gorshkov, ${ }^{1}$ David Patterson, ${ }^{1}$ Alexander S. Zibrov, ${ }^{1}$ John M. Doyle, ${ }^{1}$ \\ Mikhail D. Lukin, ${ }^{1}$ and Mara G. Prentiss ${ }^{1}$ \\ ${ }^{1}$ Harvard-MIT Center for Ultracold Atoms, Department of Physics, Harvard University, 17 Oxford Street, Cambridge, \\ Massachusetts 02138, USA \\ ${ }^{2}$ Joint Quantum Institute, Department of Physics, University of Maryland, College Park, Maryland 20742, USA
}

(Received 9 May 2008; published 6 January 2009)

\begin{abstract}
We demonstrate that buffer-gas cooling combined with laser ablation can be used to create coherent optical media with high optical depth and low Doppler broadening that offers metastable states with low collisional and motional decoherence. Demonstration of this generic technique opens pathways to coherent optics with a large variety of atoms and molecules. We use helium buffer gas to cool ${ }^{87} \mathrm{Rb}$ atoms to below $7 \mathrm{~K}$ and slow atom diffusion to the walls. Electromagnetically induced transparency in this medium allows for 50\% transmission in a medium with initial optical depth $D>70$ and for slow pulse propagation with large delaybandwidth products. In the high- $D$ regime, we observe high-contrast spectrum oscillations due to efficient four-wave mixing.
\end{abstract}

DOI: 10.1103/PhysRevA.79.013806

PACS number(s): 42.50.Gy, 42.50.Ex, 42.65.Ky

Greatly facilitated by the advent of electromagnetically induced transparency (EIT) [1,2], coherent optical atomic media have been widely used in quantum and nonlinear optics $[3,4]$, as well as in high-precision measurements [5]. For example, they underlie few-photon nonlinear optics [3] and resonant enhancement of wave mixing [4]. In addition, motivated by ideas from quantum information science, EIT is now widely used [6-13] in applications to quantum memories $[14,15]$ and to long-distance quantum communication [16]. Practical use of these applications would be greatly facilitated by more scalable simpler technologies that can simultaneously achieve high optical depth $D$ and long lifetimes for metastable-state coherences. For example, in quantum memories, $D$ and decoherence rates determine efficiency and memory time, respectively [14]. However, the two currently available atomic vapor systems-hot atoms in vapor cells $[6,7]$ and cold atoms in magneto-optical traps (MOTs) [8-12] - typically operate at $D \leqq 10$ and suffer from relatively fast decoherence of hyperfine sublevels. Although recently several experimental systems, such as high-aspectratio MOTs [17] and cavity-enhanced MOTs [13], have been shown to exhibit superior performance, improving the scalability of these systems and extending them to other atomic or molecular species is highly nontrivial due to restrictions imposed by laser cooling.

In this paper, we integrate buffer-gas cooling and laser ablation to demonstrate a simple approach to producing coherent optically dense media. Using $\mathrm{Rb}$ as an example, we achieve high $D(>70)$ combined with a low ground-state decoherence rate by cooling $\mathrm{Rb}$ vapor to below $7 \mathrm{~K}$ using a He buffer gas. This system combines the fundamental advantages of MOT systems (slow atoms with small Doppler broadening) with large volumes and simplicity characteristic of hot vapor cells. This out-of-equilibrium thermal system offers high-density metallic gases at temperatures hundreds or thousands of times colder than the boiling point of the metal. Our work is the first application of buffer-gas-cooled media to coherent quantum optics. In particular, EIT increases peak transmission in our system by a factor of $\sim \exp (70)$, while maintaining ground hyperfine decoherence rates on the order of $10 \mathrm{kHz}$. We also observe parametric four-wave mixing processes [18] and the resultant splitting of the EIT transmission peak into several peaks. Finally, we demonstrate slow pulse propagation with large delaybandwidth products.

Our cryostat system used liquid $\mathrm{He}$ to cool to $4.2 \mathrm{~K}$ a $2 \mathrm{~cm} \times 2 \mathrm{~cm} \times 2 \mathrm{~cm} \mathrm{Al}$ cell filled with He buffer gas and surrounded by Helmholtz coils to cancel stray magnetic fields (Fig. 1). Inside the cell, a compressed $\mathrm{RbCl}$ pillet was mounted as a target for laser ablation. It yielded $\mathrm{Rb}$ vapor when bombarded by a 532-nm pulsed YAG laser. Ablation pulses with typical energies of $10 \mathrm{~mJ}$ and typical durations of $3-5$ ns were used. Once generated, the hot $\mathrm{Rb}$ vapor quickly expanded until it filled the whole cell. After the ablation pulse, collisions with He cooled the Rb down to below $7 \mathrm{~K}$ in less than $1 \mathrm{~ms}$. Typically, 40 mTorr of cold He buffer gas was used (as measured by a room-temperature pressure gauge). We note that the entire cryostat system is compact (30 cm tall and $20 \mathrm{~cm}$ diameter round), reliable, and inexpensive to operate, using about 10 liters of liquid helium a day, including cooling the unit down from $300 \mathrm{~K}$ to $4 \mathrm{~K}$. We

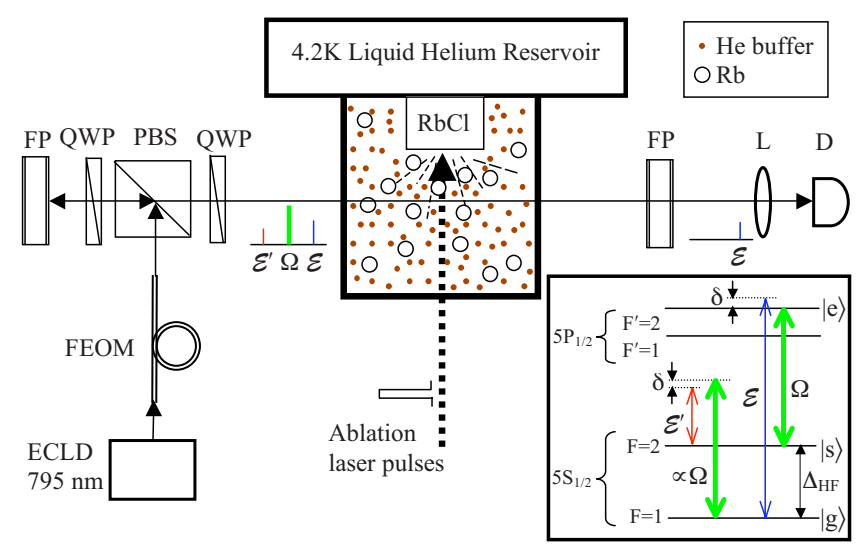

FIG. 1. (Color online) Experimental setup (PBS, polarizing beam splitter; QWP, quarter-wave plate; $L$, lens; $D$, photodiode detector). The inset shows the ${ }^{87} \mathrm{Rb}$ level structure. 

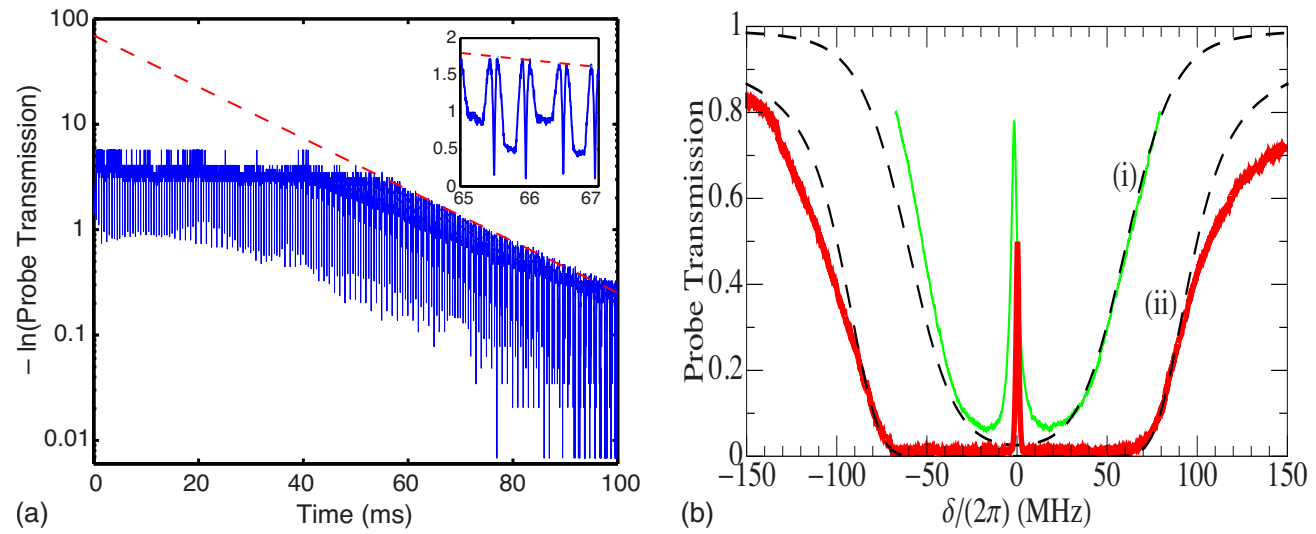

FIG. 2. (Color online) $D$ estimates in the presence of EIT. (a) Solid blue line is the negative logarithm of probe transmission as a function of time, as the probe frequency is scanned back and forth through two-photon resonance resulting in the sharp EIT dips. Red dashed line is an exponential fit to the background optical depth. The inset zooms in on the plot around $t=66 \mathrm{~ms}$. (b) Probe transmission (solid lines) at two different $D$ 's exhibiting an EIT peak on the background of a Doppler-broadened single-photon absorption dip. Dashed curves are theoretical fits to the single-photon absorption dip.

study EIT on the $\mathrm{Rb} D 1$ line $(\lambda=795 \mathrm{~nm})$ by phase modulating the light from an external-cavity diode laser (ECLD) using a fiber electro-optical modulator (FEOM) operating at the ground-state hyperfine splitting of ${ }^{87} \mathrm{Rb} \quad \Delta_{\mathrm{HF}}$ $\approx(2 \pi) 6.8 \mathrm{GHz}$. As shown in the inset of Fig. 1, the laser carrier frequency that is resonant with the $F=2 \rightarrow F^{\prime}=2$ transition played the role of the control field with Rabi frequency $\Omega$. The high-frequency modulation sideband, tuned near the $F=1 \rightarrow F^{\prime}=2$ transition with two-photon detuning $\delta$, played the role of the signal with amplitude $\mathcal{E}$. A Fabry-Perot (FP) étalon was used to block the control and the low-frequency off-resonant sideband $\mathcal{E}^{\prime}$ after the cell. For the EIT observation (but not for the four-wave mixing and slow light measurements), a second FP étalon before the cell was used to attenuate the off-resonant sideband intensity by a factor of $\approx 1 / 5$. All three optical fields were $\sigma^{+}$polarized. The physics was dominated by the $\Lambda$ system formed by $|g\rangle=\mid F=1, m$ $=1\rangle,|s\rangle=|F=2, m=1\rangle$, and $|e\rangle=\left|F^{\prime}=2, m=2\right\rangle$, which we used for modeling.

The optical depth $D$ is defined so that if we were to turn off the control field after optically pumping the atoms, the intensity of a vanishingly weak resonant probe would be attenuated by $\exp (-D)$ after passing through the medium. In the experiment, however, a continuous-wave $(\mathrm{cw})$ control field $(60 \mu \mathrm{W}, \approx 0.4 \mathrm{~mm}$ diameter) was used to provide continuous optical pumping. At the same time, two-photon detuning $\delta$ of a weak probe $(0.5 \mu \mathrm{W}, \approx 0.4 \mathrm{~mm}$ diameter $)$ was continuously scanned back and forth through two-photon resonance $(\delta=0)$ at a rate of $(2 \pi) 300 \mathrm{MHz} / \mathrm{ms}$ using a triangle wave. The measured probe transmission, thus, exhibited EIT peaks every $0.5 \mathrm{~ms}$ as a function of time while showing $\approx \exp (-D)$ in between the EIT peaks. To extract $D$, we plot in Fig. 2(a) the negative logarithm of probe transmission as a function of time (solid blue line), in which EIT peaks show up as dips and $D$ (red dashed line) can be read out between the peaks, as shown in the inset. After the ablation pulse (at time $t=0$ ), diffusion of $\mathrm{Rb}$ through the $\mathrm{He}$ buffer gas and subsequent absorption of $\mathrm{Rb}$ onto the cold cell walls reduce the density of $\mathrm{Rb}$ (and hence $D$ ). For $t$ $<50 \mathrm{~ms}, D$ is so large that the transmitted signal is com- pletely immersed in the noise background, making direct measurement of $D$ impossible. At larger $t$, however, $D$ becomes measurable and follows an exponential $D$ $\approx 75 \exp [-t /(17.5 \mathrm{~ms})]$ (red dashed line) [19]. Extrapolating this fit toward $t=0$, we obtain $D \approx 75$ near $t=0$. At these early times, EIT peaks show about 50\% transmission. To support the extrapolation of the fit to $t<50 \mathrm{~ms}$, we performed an analogous measurement on ${ }^{85} \mathrm{Rb}(2 \%$ abundance in our sample) and observed exponential behavior for $t$ $<50 \mathrm{~ms}$ (see also Ref. [20]).

In Fig. 2(b), we give an independent demonstration that high optical depths were indeed obtained and exhibit typical narrow and tall EIT two-photon resonances. The two solid lines show the probe transmission spectrum at two different $D$ 's for the same control and probe intensities as in Fig. 2(a). The single-photon Doppler-broadened resonances were fit to the negative exponential (dashed curve) of a Voigt profile made up from a convolution of a Gaussian with a full width at half maximum (FWHM) of $\Delta_{D}$ and a Lorentzian with natural FWHM of $2 \gamma=(2 \pi) 5.7 \mathrm{MHz}$. For the low- $D$ curve (i), $\Delta_{D}$ and $D$ were varied to find $D \approx 3.7$ and $\Delta_{D}$ $=(2 \pi) 75 \mathrm{MHz}$ (corresponding to an effective temperature of $7 \mathrm{~K}$ ). Taking this $\Delta_{D}$, which we will also use for the rest of the paper, and adjusting $D$ to match the flat part of the high- $D$ spectrum (ii), we obtain $D \approx 35$. We also note a narrow $[(2 \pi) 1.8 \mathrm{MHz}]$ and high-contrast $(50 \%$ transmission) EIT two-photon resonance observed in the high- $D$ curve (ii).

At high optical depths, the observed EIT transmission peak exhibits oscillations whose number increases as the optical depth increases. In Fig. 3(a), we show the probe transmission for a fixed $D$ and for different control powers. As explained below, the observed oscillations correspond to interference fringes between the directly transmitted probe output and a contribution due to four-wave mixing. Modulation is caused by the dispersion associated with EIT.

To analyze such effects quantitatively, we include the effects of four-wave mixing by following the analysis in Refs. [21-23]. The use of Floquet theory to include the far-offresonant couplings to order $1 / \Delta_{\mathrm{HF}}$ yields an effective Rabi frequency $\propto 1 / \Delta_{\mathrm{HF}}$ coupling $|g\rangle$ and $|s\rangle$. We use the master 
(a) Experiment
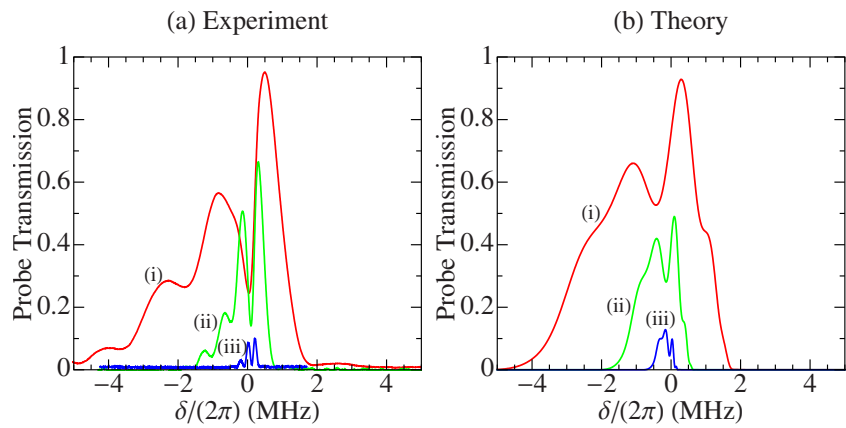

FIG. 3. (Color online) Interference fringes in the EIT spectrum caused by four-wave mixing. Control field powers are (i) $0.58 \mathrm{~mW}$, (ii) $0.21 \mathrm{~mW}$, and (iii) $0.077 \mathrm{~mW}$, with beam diameter of $0.4 \mathrm{~mm}$ (the latter deduced from the Rabi frequency fit). (a) Experiment. Since the absolute value of $\delta$ was not calibrated experimentally, $\delta$ $=0$ is chosen to approximately match the theory. (b) Theory.

equation to compute the polarizations driving $\mathcal{E}$ and $\mathcal{E}^{\prime}$ to linear order in $\mathcal{E}$ and $\mathcal{E}^{\prime}$. Ignoring the small Stark shift of $|g\rangle \propto|\Omega|^{2} / \Delta_{\mathrm{HF}}$ and integrating over the single-photon Doppler shift $\Delta$, we obtain steady-state propagation equations for $\mathcal{E}$ and $\mathcal{E}^{\prime}$ :

$$
\begin{gathered}
\partial_{z}\left[\begin{array}{c}
\mathcal{E}(z, \delta) \\
\mathcal{E}^{\prime *}(z, \delta)
\end{array}\right]=\frac{1}{L} M(\delta)\left[\begin{array}{c}
\mathcal{E}(z, \delta) \\
\mathcal{E}^{\prime *}(z, \delta)
\end{array}\right], \\
M(\delta)=\int_{-\infty}^{\infty} d \Delta \frac{e^{-\left(\Delta-\Delta_{c}\right)^{2} / 2 \sigma^{2}}}{\sqrt{2 \pi} \sigma} i \frac{d \gamma}{F}\left[\begin{array}{cc}
\delta+i \gamma_{0} & -\frac{\Omega^{2}}{\Delta_{\mathrm{HF}}} \\
\frac{\Omega^{* 2}}{\Delta_{\mathrm{HF}}} & 0
\end{array}\right] .
\end{gathered}
$$

Here $F=|\Omega|^{2}-(\delta+\Delta+i \gamma)\left(\delta+i \gamma_{0}\right), L$ is the medium length, $\gamma_{0}$ is the decay rate of the $|s\rangle-|g\rangle$ coherence, $\sigma$ $=\Delta_{D} /(2 \sqrt{2 \ln 2})$ is the standard deviation of the Doppler profile, and $d=\frac{3}{8 \pi} \lambda^{2} L \frac{N}{V}$ would have been half of the resonant optical depth if there were no Doppler broadening [i.e., $|e(L, 0)|^{2}=\exp (-2 d)|e(0,0)|^{2}$ for $\Delta_{D} \rightarrow 0$ and $\left.\Omega=0\right]$. $N / V$ is the density of atoms in state $|g\rangle$. For our values of $\gamma$ and $\Delta_{D}$, $d=4.6 D$. We have also allowed for a possible deviation $\Delta_{c}$ of the control field from resonance.

Given $\mathcal{E}(0, \delta)=-\mathcal{E}^{\prime}(0, \delta)=1$, we solve Eq. (1) for probe transmission $|\mathcal{E}(L, \delta)|^{2}$ by exponentiating $M(\delta)$. The result is shown in Fig. 3(b). For curve (i), $\Omega=(2 \pi) 15 \mathrm{MHz}$ and $D$ $=100$ were chosen to approximately reproduce the total peak width and the fringe period. For (ii) and (iii), $\Omega$ was computed from the known intensity ratios. $\gamma_{0}=(2 \pi) 30 \mathrm{kHz}$ was adjusted to approximately reproduce the relative amplitudes of the three curves. $\Delta_{c}=(2 \pi) 40 \mathrm{MHz}$ was chosen to reproduce the left-right asymmetry of the data.

To get insight into the nature of the oscillations, we note that $M_{11}(\delta)$ describes EIT propagation, while the offdiagonal elements of $M$ are the parametric coupling coefficients giving four-wave mixing. Large off-diagonal matrix elements would result in exponential growth (gain). In the present experiment, however, the four-wave mixing terms are small due to the large $\Delta_{\mathrm{HF}}$, so that $\exp [M(\delta)]$ can be expanded to first order in $1 / \Delta_{\mathrm{HF}}$ to give

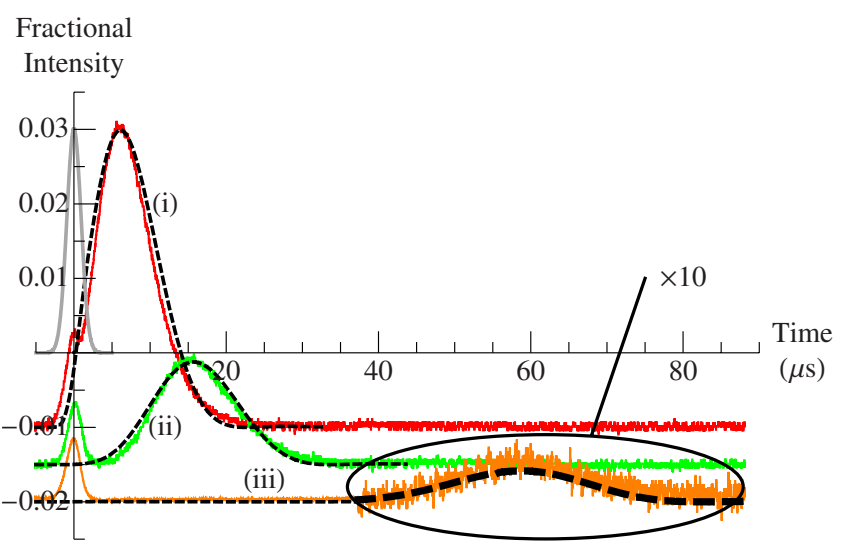

FIG. 4. (Color online) Pulse propagation through EIT medium at three different optical depths increasing from (i) to (iii). Intensity of 1 corresponds to the maximum intensity of the incident pulse, a down-scaled version of which (gray) is centered at time $=0$. The three experimental data sets (solid lines) and the simulations (dashed lines) are shifted vertically relative to each other for easier viewing. The circled piece of data set (iii) and the corresponding theory are magnified by a factor of 10 for easier viewing. Control field of $400 \mu \mathrm{W}$ and $3.2 \mathrm{~mm}$ diameter (the latter deduced from the Rabi frequency fit) is used. The absolute delays are (i) $6 \mu \mathrm{s}$, (ii) $16 \mu \mathrm{s}$, and (iii) $59 \mu \mathrm{s}$, yielding delay-bandwidth products (where bandwidth is given by the output pulse) of (i) 0.7 , (ii) 1.2 , and (iii) 2.9. From the theoretical fit, the fraction of energy transmitted is: (i) $18 \%$, (ii) $8 \%$, and (iii) $0.4 \%$.

$$
|\mathcal{E}(L, \delta)|^{2} \approx\left|e^{M_{11}(\delta)}+\frac{\Omega^{2}}{\Delta_{\mathrm{HF}}\left(\delta+i \gamma_{0}\right)}\left(e^{M_{11}(\delta)}-1\right)\right|^{2} .
$$

Thus, the probe spectrum is an interference of EIT $\left[\exp \left(M_{11}\right)\right]$ and four-wave mixing $\left(\propto 1 / \Delta_{\mathrm{HF}}\right)$. Since the phase of the EIT term is $\approx \delta /\left(v_{g} / L\right)\left[v_{g}=L \Omega^{2} /(\gamma d)\right.$ is the group velocity], while the phase of the four-wave mixing term is approximately $\delta$-independent (except for a sign change at $\delta$ $=0$ ), the fringes have period $2 \pi v_{g} / L$. Notice that it is the homogeneous optical depth $2 d$ (not $D$ ) that determines $v_{g}$ [24].

The demonstrated interference fringes confirm that the medium combines high optical depths and long coherence times because lower $D$ and higher $\gamma_{0}$ give theoretical spectra inconsistent with the data. In particular, smaller $D$ increases the fringe period, while larger $\gamma_{0}$ reduces transmission and increases the ratio of the amplitudes of the three curves. The mismatch between experimental and theoretical curves most likely arises from features not included in our simple model. In particular, velocity-changing coherence-preserving collisions alleviate the effects of Doppler broadening [25] and may explain the high contrast of the experimentally observed fringes.

We now consider propagation of a probe pulse with intensity envelope FWHM $T=2.2 \mu$ s in the presence of a cw control field. The fraction of intensity transmitted is shown in Fig. 4 with the solid lines showing the experiment and the dashed lines showing the excellent theoretical fit (see below). The three curves, corresponding to different $D$ 's increasing 
from (i) to (iii), exhibit large fractional delays that increase with $D$.

To model the data, we set $\Delta_{c}=0$ and determined the Rabi frequency for all three plots in the figure by fitting the data in (i) using $D$ and $\Omega$ as free parameters, resulting in $D=7.5$ and $\Omega=(2 \pi) 1.5 \mathrm{MHz}$. This fit was also used to determine the vertical scale for all three curves, because the absolute intensities were not measured. For plots (ii) and (iii) $D$ was used as a free parameter to reproduce the delays, giving a $D$ of 16 for (ii) and a $D$ of 59 for (iii). Finally, $\gamma_{0}=(2 \pi) 5 \mathrm{kHz}$ was adjusted to reproduce the ratio of the maximum intensities of (i) and (ii). The measured fractional delays are very well approximated by $L /\left(v_{g} T\right)$, where the group velocity $v_{g}$ $=L \Omega^{2} /(\gamma d)$ is given in terms of homogeneously broadened optical depth $2 d$ [24]. The initial short pulse visible on the experimental data is $\approx 1 \%$ of the transmitted off-resonant sideband pulse, which leaked through the FP étalon after the cell.

In summary, we combined buffer-gas cooling with laser ablation to obtain a simple method to generate coherent optical media. As an example of this method, we cooled ${ }^{87} \mathrm{Rb}$ by He buffer gas to below $7 \mathrm{~K}$ to produce an optically thick medium with low ground-state decoherence, excellent EIT, strong enhancement of nonlinearities, and large fractional delays in slow light propagation. By optimizing such factors as buffer-gas pressure, cell geometry, magnetic shielding, and the ablation procedure, we expect to increase $D$, decrease decoherence, and tailor the system to specific applications. Our system combines the slow atomic motion and good coherence properties of MOT systems with the simplicity and large atomic volume of vapor cell systems. Moreover, our system can be used to produce coherent optical media of other atoms, molecules, or their combinations by simply replacing the source. All these features distinguish our method and this particular implementation of it as promising candidates for the use in nonlinear and quantum optics as well as in precision measurements. In particular, applications to delay lines, quantum memories, and sources of single or paired photons can be foreseen.

We thank E. N. Fortson for lending us a diode laser, A. A. Zibrov for help in building the setup, and M. Parsons, W. Campbell, E. Tsikata, M. Hummon, and M. Hohensee for discussions. This work was supported by NSF CUA and GRFP, DARPA, NSF Grant No. PHY-0757157.
[1] S. E. Harris, Phys. Today 50 (7), 36 (1997).

[2] M. Fleischhauer, A. Imamoglu, and J. P. Marangos, Rev. Mod. Phys. 77, 633 (2005).

[3] A. Imamoglu, H. Schmidt, G. Woods, and M. Deutsch, Phys. Rev. Lett. 79, 1467 (1997); S. E. Harris and Y. Yamamoto, ibid. 81, 3611 (1998); T. Hong, ibid. 90, 183901 (2003).

[4] P. R. Hemmer et al., Opt. Lett. 20, 982 (1995); S. E. Harris et al., Philos. Trans. R. Soc. London, Ser. A 355, 2291 (1997).

[5] I. K. Kominis et al., Nature (London) 422, 596 (2003); T. Hong, C. Cramer, W. Nagourney, and E. N. Fortson, Phys. Rev. Lett. 94, 050801 (2005); T. W. Kornack, R. K. Ghosh, and M. V. Romalis, ibid. 95, 230801 (2005).

[6] M. D. Eisaman et al., Nature (London) 438, 837 (2005).

[7] J. Appel, E. Figueroa, D. Korystov, M. Lobino, and A. I. Lvovsky, Phys. Rev. Lett. 100, 093602 (2008).

[8] T. Chanelière et al., Nature (London) 438, 833 (2005).

[9] K. S. Choi et al., Nature (London) 452, 67 (2008).

[10] K. Honda, D. Akamatsu, M. Arikawa, Y. Yokoi, K. Akiba, S. Nagatsuka, T. Tanimura, A. Furusawa, and M. Kozuma, Phys. Rev. Lett. 100, 093601 (2008).

[11] P. Kolchin, S. Du, C. Belthangady, G. Y. Yin, and S. E. Harris, Phys. Rev. Lett. 97, 113602 (2006).

[12] Z.-S. Yuan, Y. A. Chen, S. Chen, B. Zhao, M. Koch, T. Strassel, Y. Zhao, G. J. Zhu, J. Schmiedmayer, and J. W. Pan, Phys. Rev. Lett. 98, 180503 (2007).

[13] J. Simon, H. Tanji, J. K. Thompson, and V. Vuletić, Phys. Rev. Lett. 98, 183601 (2007).

[14] M. D. Lukin, Rev. Mod. Phys. 75, 457 (2003).

[15] M. Fleischhauer, and M. D. Lukin, Phys. Rev. Lett. 84, 5094 (2000); Phys. Rev. A 65, 022314 (2002).
[16] L. M. Duan et al., Nature (London) 414, 413 (2001).

[17] M. Vengalattore and M. Prentiss, Phys. Rev. Lett. 95, 243601 (2005); S. Du, P. Kolchin, C. Belthangady, G. Y. Yin, and S. E. Harris, ibid. 100, 183603 (2008).

[18] E. E. Mikhailov, Yu. V. Rostovtsev, and G. R. Welch, J. Mod. Opt. 50, 2645 (2003); V. Boyer, C. F. McCormick, E. Arimondo, and P. D. Lett, Phys. Rev. Lett. 99, 143601 (2007); H. Kang, G. Hernandez, and Y. Zhu, Phys. Rev. A 70, 061804 (2004); V. Wong, R. S. Bennink, A. M. Marino, R. W. Boyd, C. R. Stroud, and F. A. Narducci, ibid. 70, 053811 (2004); K. Harada, T. Kanbashi, M. Mitsunaga, and K. Motomura, ibid. 73, 013807 (2006); G. S. Agarwal, T. N. Dey, and D. J. Gauthier, ibid. 74, 043805 (2006).

[19] Due to the variation of $D$ from one ablation to another, $D$ is used below as a fit parameter.

[20] A. O. Sushkov and D. Budker, Phys. Rev. A 77, 042707 (2008).

[21] M. D. Lukin, M. Fleischhauer, A. S. Zibrov, H. G. Robinson, V. L. Velichansky, L. Hollberg, and M. O. Scully, Phys. Rev. Lett. 79, 2959 (1997)

[22] M. D. Lukin, P. R. Hemmer, M. Loffler, and M. O. Scully, Phys. Rev. Lett. 81, 2675 (1998).

[23] M. D. Lukin, A. B. Matsko, M. Fleischhauer, and M. O Scully, Phys. Rev. Lett. 82, 1847 (1999).

[24] M. M. Kash, V. A. Sautenkov, A. S. Zibrov, L. Hollberg, G. R. Welch, M. D. Lukin, Y. Rostovtsev, E. S. Fry, and M. O. Scully, Phys. Rev. Lett. 82, 5229 (1999).

[25] M. V. Pack, R. M. Camacho, and J. C. Howell, Phys. Rev. A 76, 013801 (2007). 